\title{
Lipid pickup and delivery
}

\author{
Howard Riezman and Gerrit van Meer \\ Intracellular organelles have specific lipid and protein compositions. Although great progress has been made \\ concerning the mechanisms that govern the transport of proteins between organelles, little information is available \\ about the mechanisms of inter-organellar lipid transport. New work identifies a protein that specifically transports \\ ceramide, a precursor of sphingolipid synthesis, between intracellular organelles.
}

Sphingolipids and the intermediates in their biosynthesis are important cellular components, not only because of their contribution to membrane lipid bilayers, but also through their role in the formation of membrane microdomains, termed ' $r a f t s$ ' ${ }^{1}$, and as signalling molecules. Therefore, sphingolipid synthesis and compartmentalization of the intermediates in this process must be tightly regulated. The initial steps resulting in ceramide synthesis occur in the endoplasmic reticulum. A pair of homologous genes, LAG1 and $L A C 1$, has been identified in the budding yeast Saccharomyces cerevisiae and is required for this step ${ }^{2,3}$. After trans- $\Delta 4$-unsaturation of the sphingoid chain by the DES1 desaturase ${ }^{4}$, conversion of ceramide into sphingomyelin, the next enzymatic step, occurs in the Golgi compartment.

Lipids are transported along with proteins between organelles by vesicular traffic, but lipids can also be delivered more directly via non-vesicular pathways. In Nature, Hanada and colleagues ${ }^{5}$ characterize a protein that extracts ceramide from the endoplasmic reticulum and delivers it specifically to the Golgi apparatus for conversion to sphingomyelin. Previously, two approaches have shed light on the delivery of ceramide from the endoplasmic reticulum to the Golgi compartment. One of these is a biochemical approach used both in mammalian cells ${ }^{6}$ and in yeast ${ }^{7}$. In both systems it was shown that transport of ceramide from the endoplasmic reticulum to the Golgi can occur through a non-vesicular pathway. Reconstitution of this pathway in the test tube showed that cytosolic proteins are required for this transport. In yeast, it was shown that membrane-membrane contact, presumably between endoplasmic reticulum and the Golgi compartments, is also required for efficient non-vesicular transport. The

Howard Riezman is in the Department of

Biochemistry, University of Geneva, $\mathrm{CH}-1211$

Geneva 4, Switzerland. Gerrit van Meer is in the Institute of Biomembranes, Utrecht University, 3584 CH, Utrecht, The Netherlands.

e-mail:howard.riezman@biochem.unige.chor

g.vanmeer@chem.uu.nl

Domains of CERT

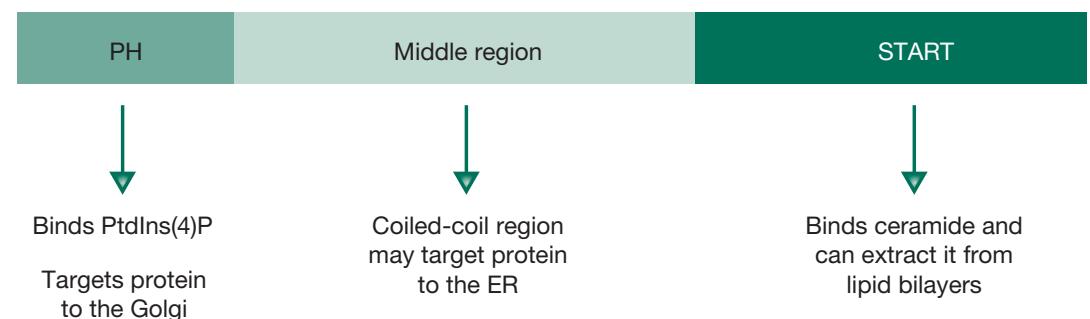

Figure 1 CERT domains function in ceramide transport. The middle region of CERT may contain information targeting the protein to the endoplasmic reticulum, where the START domain can bind to and extract ceramide from the cytosolic leaflet of the membrane. CERT would then be directed by its pleckstrin homology $(\mathrm{PH})$ domain, which binds to Ptdlns(4)P, to the Golgi apparatus. CERT would then release ceramide into the cytosolic leaflet of the Golgi membrane.

other approach, used by Hanada and colleagues, is genetic ${ }^{8}$. They isolated mammalian cell mutants that were resistant to lysenin, a toxic compound that binds specifically to sphingomyelin on the cell surface. One of the resistant clones, LY-A, turned out to be deficient in a cytosolic factor that is required for the transport of ceramide from the endoplasmic reticulum to the site of sphingomyelin synthesis in the Golgi compartment.

Sphingolipids associate with cholesterol to form raft microdomains in the plasma membrane. LY-A cells, which are deficient in cellsurface sphingomyelin, probably have less stable rafts because the cells are hypersensitive to methyl- $\beta$-cyclodextrin, an agent that extracts cholesterol from membranes and is therefore toxic. In their current study, Hanada and colleagues used this interesting property of LY-A cells to select a clone from LY-A cells transfected with a human cDNA library that is protected against the toxic effects of cyclodextrin treatment. They identified a multifunctional protein they termed CERT. CERT was originally studied as a protein called GPBP, for Goodpasture antigenbinding protein. It was found to bind the carboxyl terminus of human collagen $\alpha 3$ in vitro ${ }^{9}$, although this is unlikely to be related to a physiological role because CERT (GPBP) is located in the cytosol and collagen is extracellular. This new study suggests that instead, the physiological role of CERT is in ceramide transport from the endoplasmic reticulum to the Golgi compartment. CERT is mutated in the LY-A mutant cell line and wild-type CERT can restore activity to LY-A mutant cytosol in the in vitro assay for non-vesicular ceramide transport ${ }^{5}$.

Hanada and colleagues have shown that CERT has several interesting properties that are necessary for ceramide transport from the endoplasmic reticulum to the Golgi compartment. CERT is probably composed of three domains (Fig. 1). The amino-terminal 120 amino acids contain a pleckstrin homology domain that binds to phosphatidylinositol-4-phosphate $(\operatorname{PtdIns}(4) \mathrm{P})^{10}$, the middle of the protein has coiled-coil motifs that could be involved in self-assembly, and the carboxy-terminal 230 amino acids contain a START domain. The START domain of CERT recognizes ceramide and is required and sufficient for ceramide extraction. Although CERT can extract ceramide from liposomes of defined composition, it does not extract cholesterol, sphingosine, sphingomyelin or phosphatidylcholine ${ }^{5}$, even though other START domains recognize specific lipids including cholesterol ${ }^{11,12}$ or phosphatidylcholine ${ }^{13}$. Furthermore, CERT can extract ceramide from an intact organelle: the endoplasmic reticulum. CERT contains a consensus protein sequence that has been 


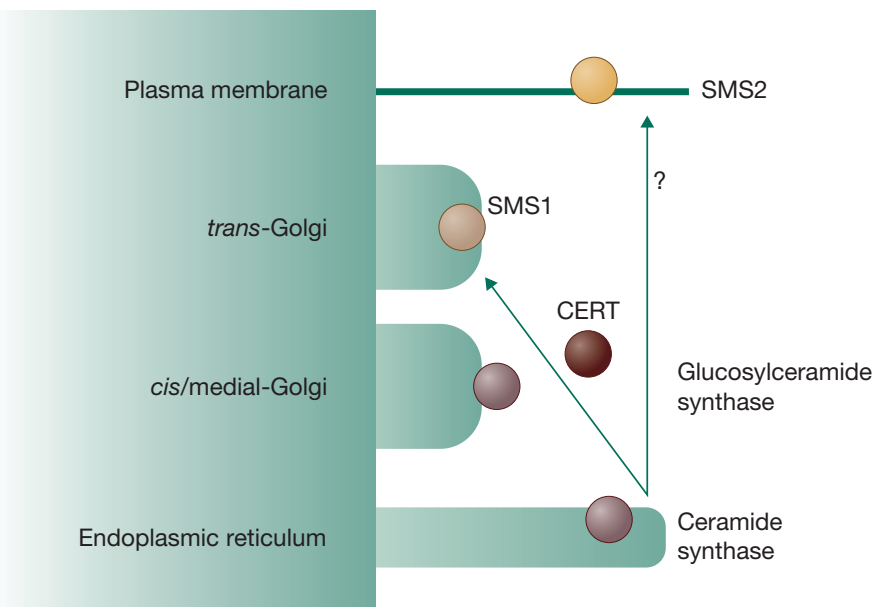

Figure 2 Model arrangement for various enzymes of ceramide synthesis and transport. Ceramide is synthesized in the endoplasmic reticulum, from where it is delivered by CERT to the transGolgi and SMS1. CERT is not required to provide ceramide for glucosylceramide synthase in the cis-Golgi compartment. Another sphingomyelin synthase, SMS2, is localized to the plasma membrane. The ceramide for this reaction may result from sphingomyelin hydrolysis in the plasma membrane by sphingomyelinases involved in signal transduction, but as Ptdlns(4)P is also present in the plasma membrane, a role for CERT cannot be excluded.

implicated in targeting cytosolic proteins to the endoplasmic reticulum, although this domain in CERT has not been shown to function in targeting CERT to the endoplasmic reticulum. Finally, the pleckstrin homology domain of CERT has been shown to actually target the protein to the Golgi compartment where the sphingomyelin synthase is located. Importantly, the mutation of CERT in LY-A cells (G67E) lies in this domain and disrupts the ability of CERT to associate with the Golgi compartment. These results imply that the ability of CERT to associate with the Golgi is important for its function in ceramide transport. Interestingly, a mutation in a phosphatidylinositol-4-OH kinase also confers a defect in non-vesicular aminophospholipid transport in yeast ${ }^{14}$, suggesting that targeting of phospholipid carriers to membranes by PtsIns(4)P may be a general principle in non-vesicular lipid transport. Thus, CERT has all of the properties necessary to pick up ceramide from the endoplasmic reticulum and deliver it to the Golgi.

Previously, it has been unclear exactly where sphingomyelin synthase is located in the Golgi stack. Recent work identifying the elusive sphingomyelin synthase has now provided an answer to this question. Huitema and colleagues ${ }^{15}$ have identified two human sphingomyelin synthases (SMSs), of which SMS1 fulfills many criteria to be the common Golgi sphingomyelin synthase. SMS1 is a
Golgi protein with a relative molecular mass $\left(M_{\mathrm{r}}\right)$ of 49,000 . It has 6 predicted transmembrane domains with the active centre on the lumenal side. It synthesizes sphingomyelin by transferring the phosphocholine headgroup of phosphatidylcholine to ceramide. By immunofluorescence microscopy, SMS1 colocalized with sialyltransferase, a marker of trans-Golgi cisternae. This suggests the possibility that direct ER-trans-Golgi membrane contacts, which have been visualized previously ${ }^{16}$, may facilitate the process by which CERT transfers ceramide between the two membranes. Remarkably, the mutation of CERT in LY-A cells that inactivated sphingomyelin synthesis did not affect the synthesis of glucosylceramide, which occurs in the cisand medial-cisternae of the Golgi. So, either the mutated CERT still docked and delivered ceramide to the cis- and medial-Golgi, but not to the trans-Golgi network, or alternatively ceramide reaches the cis- and medial-Golgi by another mechanism (Fig. 2).

Besides being transported from the endoplasmic reticulum to the plasma membrane, lipids can also be internalized and delivered to the endoplasmic reticulum from the plasma membrane. A product of the sphingomyelin synthase reaction is diacylglycerol. Similarly to ceramide, diacylglycerol is a signalling lipid, and an important question is how cells handle this potentially dangerous mediator. Interestingly, CERT can extract (albeit inefficiently) diacylglycerol, which is after all structurally related to ceramide. It is possible that CERT transfers diacylglycerol back to the endoplasmic reticulum where it functions as a precursor for glycerophospholipid synthesis.

In addition to transporting ceramide between the endoplasmic reticulum and Golgi, it is possible that CERT also transports ceramide between other intracellular sites. Ceramide and diacylglycerol also exist at the plasma membrane, where they are generated in the course of signalling events by sphingomyelinases and phospholipases $\mathrm{C}$, respectively. A second human sphingomyelin synthase, SMS2, was localized to the plasma membrane and, similarly to SMS1, SMS2 was found capable of catalysing the conversion of phosphatidylcholine and ceramide to sphingomyelin and diacylglycerol, as well as the reverse reaction. It remains an enigma how ceramide and diacylglycerol are removed from the plasma membrane. Surprisingly, a lipid transfer protein, the phosphatidylinositol transfer protein $\beta$, which is capable of binding sphingomyelin and resides on the cytosolic surface of the Golgi, was found to stimulate the (re)synthesis of sphingomyelin from plasma membrane ceramide ${ }^{17}$. Whether this protein interacts directly with SMS2 or, similarly to CERT, transfers lipids through the cytosol, is one of the important open questions in this blooming field. Another intriguing question is to understand whether and how CERT or other lipid transport proteins use membrane contact sites to facilitate lipid transport between organelles.

1. Simons, K.\& Ikonen, E. Nature 387, 569-572 (1997).

2. Guillas, I. et al. EMBO J. 20, 2655-2665 (2001).

3. Schorling, S., Vallee, B., Barz, W. P., Riezman, H. \& Oesterhelt, D. Mol. Biol. Cell 12, 3417-3427 (2001).

4. Ternes, P., Franke, S., Zahringer, U., Sperling, P. \& Heinz, E. J. Biol. Chem. 277, 25512-25518 (2002).

5. Hanada, K. et al. Nature DOI: 10.1038/nature02240 (2004).

6. Funakoshi, T., Yasuda, S., Fukasawa, M., Nishijima, M. \& Hanada, K. J. Biol. Chem. 275, 29938-29945 (2000).

7. Funato, K.\& Riezman, H. J. Cell Biol. 155, 949-959 (2001).

8. Fukasawa, M., Nishijima, M. \& Hanada, K. J. Cell Biol. 144, 673-685 (1999).

9. Raya, A. et al. J. Biol. Chem. 275, 40392-40399 (2000).

10. Levine, T. P.\& Munro, S. Curr. Biol. 12, 695-704 (2002).

11. Kallen, C. B. et al. J. Biol. Chem. 273, 26285-26288 (1998).

12. Tsujishita, Y. \& Hurley, J. H. Nature Struct. Biol. 7, 408-414 (2000).

13. Akeroyd, R., Moonen, P., Westerman, J., Puyk, W. C. \& Wirtz, K. W. Eur. J. Biochem. 114, 385-391 (1981).

14. Trotter, P. J., Wu, W. I., Pedretti, J., Yates, R. \& Voelker, D. R. J. Biol. Chem. 273, 13189-13196 (1998).

15. Huitema, K., van den Dikkenberg, J., Brouwers, J. \& Holthuis, J. EMBO J. (in the press).

16. Ladinsky, M. S., Mastronarde, D. N., McIntosh, J. R., Howell, K. E. \& Staehelin, L. A. J. Cell Biol. 144, 1135-1149 (1999).

17. Van Tiel, C. M., Luberto, C., Snoek, G. T., Hannun, Y. A. \& Wirtz, K. W. Biochem J. 346, 537-543 (2000). 